\title{
Oral Cancer Vaccine V3-OVA
}

National Cancer Institute

\section{Source}

National Cancer Institute. Oral Cancer Vaccine V3-OVA. NCI Thesaurus. Code C159540.

An orally available cancer vaccine composed of autologous ovarian cancer antigens obtained from hydrolyzed, inactivated blood and tumor tissue of patients with ovarian cancer, with potential immunostimulatory and antineoplastic activities. Upon oral administration of the oral cancer vaccine V3-OVA, the ovarian cancer antigens stimulate the immune system and activate a cytotoxic T-lymphocyte (CT L) immune response against ovarian cancer cells. 